\title{
Avaliação estética e funcional do tratamento cirúrgico do torcicolo congênito com a técnica de liberação distal do músculo esternocleidomastoideo
}

\author{
Aesthetic and functional evaluation of the surgical treatment of \\ congenital muscular torticollis applying the technique of the lower \\ liberation of the sternocleidomastoid contracture
}

Evaluación estética y funcional del tratamiento quirúrgico del tortícolis congénito con la técnica de liberación distal del músculo esternocleidomastoideo

\author{
Osmar Avanzi ${ }^{1}$ \\ Robert Meves ${ }^{2}$ \\ Maria Fernanda Silber Caffaro ${ }^{3}$ \\ Bruno César Aprile ${ }^{4}$
}

\section{RESUMO}

Objetivo: realizar uma avaliação estética e funcional do tratamento cirúrgico do torcicolo congênito por meio da técnica de liberação distal do músculo esternocleidomastoideo. Métodos: foi realizada análise cirúrgica retrospectiva em 21 pacientes portadores de torcicolo congênito submetidos à liberação distal da contratura do músculo esternocleidomastoideo no período de Setembro de 1986 a Setembro de 2003 no Departamento de Ortopedia e Traumatologia da Faculdade de Ciências Médicas da Santa Casa de São Paulo, avaliados pelo protocolo de Cheng e Tang. Resultados: a média do tempo de seguimento foi de 55 meses (variando de 6 meses a 17 anos). Este

\section{ABSTRACT}

Objective: to evaluate the treatment outcome of surgical treatment of congenital torcicollis through distal liberation of sternocleidomastoid muscle. Methods: the result of the surgical treatment of 21 patients bearing congenital muscular torticollis, submitted to the lower liberation of the sternocleidomastoid muscle's contracture, from September of 1986 through September of 2003, at the Department of Orthopedics and Traumatology of the Faculdade de Ciências Médicas of the Santa Casa de São Paulo, was retrospectively evaluated using Cheng and Tang's protocol. Results: with an average of 55 months of follow-up (ranging from 6 months to 17 years), this
\end{abstract}

\section{RESUMEN}

Objetivo: realizar una evaluación estética y funcional del tratamiento quirúrgico del tortícolis congénito por medio de la técnica de liberación distal del músculo esternocleidomastoideo. Métodos: los autores realizaron un análisis quirúrgico retrospectivo en 21 pacientes portadores del torticolis congénito. Todos fueron sometidos a la liberación distal de la contractura del músculo esternocleidomastoideo en el período de Septiembre de 1986 a Septiembre de 2003, en el Departamento de Ortopedia e Traumatologia da Faculdade de Ciências Médicas da Santa Casa de São Paulo, evaluados por el protocolo de Cheng \& Tang. Resultados: el promedio del tiempo de seguimiento fue de 55 meses (con variación de 6 meses a

\footnotetext{
Trabalho realizado pelo Grupo de Coluna do Departamento de Ortopedia e Traumatologia da Faculdade de Ciências Médicas da Santa Casa de São Paulo - FC MSCSP - São Paulo (SP), Brasil.

1 Professor Adjunto da Faculdade de Ciências Médicas da Santa Casa de São Paulo -FCMSCSP - São Paulo (SP), Brasil.

2 Professor-assistente do Departamento de Ortopedia e Traumatologia da Faculdade de Ciências Médicas da Santa Casa de São Paulo - FCMSCSP - São Paulo (SP), Brasil.

${ }^{3}$ Professor Instrutor da Faculdade de Ciências Médicas da Santa Casa de São Paulo -FCMSCSP - São Paulo (SP), Brasil.

${ }^{4}$ Estagiário do Curso de Aperfeiçoamento do Grupo de Coluna da Faculdade de Ciências Médicas da Santa Casa de São Paulo - FCMSCSP - São Paulo (SP), Brasil. 
estudo revelou que 20 pacientes (95\%) apresentaram um resultado estético-funcional bom ou excelente com poucas complicações inerentes ao procedimento. Conclusões: na presente casuística, observou-se um resultado estético-funcional satisfatório no tratamento cirúrgico do torcicolo muscular congênito.

DESCRITORES: Torcicolo/ congênito; Torcicolo/ cirurgia; Contratura; Músculo esquelético; Resultado de tratamento study revealed that 20 patients (95\%) showed good or even excellent aesthetic-functional results, with few complications due to the procedure. Conclusions: in the present experience, the surgical treatment of the congenital muscular torticollis showed good or even excellent aesthetic-functional results.

KEYWORDS: Torticollis/ congenital; Torticollis/ surgery; Contracture; Muscle, skeletal; Treatment outcome
17 años). Este estudio reveló que $20 \mathrm{pa}$ cientes $(95 \%)$ presentaron un resultado estético/funcional bueno o excelente, con pocas complicaciones inherentes al procedimiento. Conclusiones: fue observado, en la presente casuística, un resultado estéticolfuncional satisfactorio en el tratamiento quirúrgico del tortícolis muscular congénito.

DESCRIPTORES: Tortícolis/ congenita; Tortícolis/cirugía; Contractura; Músculo esquelético; Resultado de tratamiento

\section{INTRODUÇÃO}

O termo "torcicolo" foi definido por Tubby, em 1912, como uma deformidade, congênita ou adquirida, caracterizada pela inclinação da cabeça em direção ao ombro, associada à torção do pescoço e desvio da face. O torcicolo muscular congênito consiste em uma contratura fibrosa do músculo esternocleidomastoideo (ECM), relacionada à afecção primária do músculo, que é detectada no nascimento ou nas primeiras semanas de vida. Esta doença deve ser diferenciada dos outros tipos de torcicolos congênitos ou adquiridos como, por exemplo, as anomalias esqueléticas congênitas, condições pós-traumáticas, infecções, inflamações de estruturas adjacentes, condições tumorais, ocular e distonias neurológicas ${ }^{1}$.

Macdonald ${ }^{2}$ dividiu os pacientes em dois grupos: um portador de tumoração na substância do músculo e outro com o músculo contraturado ${ }^{2,3}$. Hulbert ${ }^{4}$ relatou ainda a existência de um grupo chamado "torcicolo postural", que seria composto por aqueles pacientes com deformidade, porém, sem tumoração ou contratura do ECM. Em geral, evoluem com resolução espontânea na grande maioria dos $\operatorname{casos}^{3-5}$.

A incidência de torcicolo muscular congênito varia de 0,3 a $2,0 \%{ }^{3}$, sendo a terceira anomalia ortopédica congênita mais comum, após a displasia congênita do quadril e o pé torto congênito ${ }^{6}$. Sua etiologia ainda é motivo de grande controvérsia. Hipóteses da origem desta deformidade incluem: trauma no parto, hereditariedade, mau posicionamento intrauterino, isquemia, oclusão venosa e síndrome compartimental; no entanto, todas carecem de comprovação ${ }^{1}$.

O diagnóstico do torcicolo muscular congênito é clínico, mas anormalidades esqueléticas e outras doenças capazes de reproduzir os mesmos sinais clínicos devem ser afastadas. Os achados mais frequentes são: inclinação da cabeça, limitação da rotação e flexão lateral do pescoço, uma massa e/ou cordão fibroso palpável na topografia do
ECM (Figura 1). A ultrassonografia, a tomografia computadorizada e a ressonância magnética são exames complementares que, embora pouco utilizados em nosso meio para este fim, podem auxiliar no diagnóstico ${ }^{7,8}$.

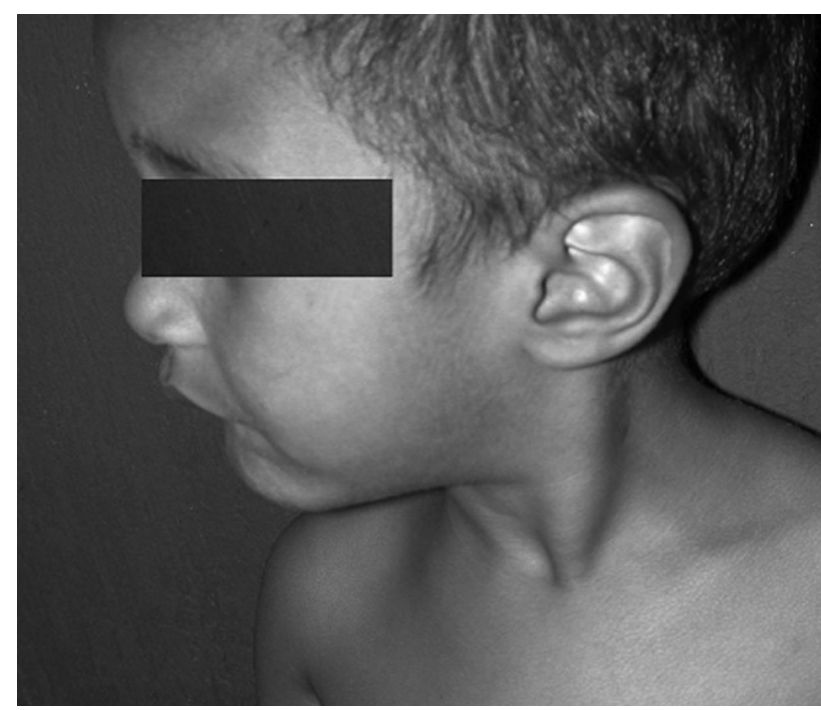

Figura 1

Fotografia ilustrando a contratura do músculo esternocleidomastoideo.

O tratamento nos primeiros meses de vida inclui observação clínica, uso de órteses, aplicação de um programa ativo de estimulação e posicionamento, alongamento manual do músculo e manipulação vigorosa do ECM contraturado. O tratamento cirúrgico, por sua vez, fica reservado aos pacientes com maior faixa etária e músculo tenso com banda de constrição; para aqueles que apresentam inclinação persistente da cabeça, com déficit de rotação e inclinação lateral passiva maior que $15^{\circ}$; ou ainda para os pacientes que não apresentaram qualquer melhora nos últimos seis meses de alongamento manual ${ }^{1,3,8,9}$. 
O tratamento cirúrgico do torcicolo muscular congênito pode ser realizado por tenotomia subcutânea, tenotomia aberta do músculo, procedimentos mioplásticos, tenotomia bipolar e ressecção radical do $\mathrm{ECM}^{1,8}$. O tratamento pós-operatório também é controverso. Alguns estudos defendem o uso de órteses ou imobilizações gessadas por um período que varia de três semanas a seis meses, enquanto outros indicam apenas a fisioterapia precoce e continuada para a reeducação postural e evitar recidivas ${ }^{1,3,5}$. Melhores resultados estéticos e da mobilidade cervical são esperados quando o tratamento cirúrgico é realizado até os 12 anos de vida, evitando, assim, sequelas como deformidade facial ou dos ossos cranianos ${ }^{10}$.

Este estudo teve como objetivo analisar o resultado estético e funcional do tratamento cirúrgico em pacientes portadores de torcicolo congênito submetidos à técnica de liberação distal do ECM aberta.

\section{MÉTODOS}

Foram avaliados 21 pacientes, sem distinção quanto ao sexo, portadores de torcicolo congênito, submetidos ao tratamento cirúrgico com utilização de técnica para a liberação distal e ressecção de cerca de 1,0 cm do ECM, de Setembro de 1986 a Setembro de 2003. O diagnóstico foi realizado pelo quadro clínico, sendo descartada qualquer outra causa possível de torcicolo. Todos os pacientes foram submetidos a radiografias e não apresentavam alterações esqueléticas na coluna cervical.

\section{Técnica cirúrgica}

Os pacientes foram submetidos à anestesia geral e, após os procedimentos de assepsia e antissepsia de rotina, foi realizada incisão transversa adjacente à clavícula para ressecção distal da contratura fibrosa do ECM. Ambas as inserções, esternal e clavicular, e contraturas adicionais de partes moles foram ressecadas quando necessário. Após sutura intradérmica, os pacientes ficaram imobilizados com colar cervical por até sete dias para posterior confecção de gesso minerva que foi mantido por cinco a seis semanas, sendo realizadas, no máximo, três cunhas corretivas seriadas no gesso. Depois de retirado o gesso, houve indicação de fisioterapia motora e exercícios domiciliares.

Em relação à avaliação pós-operatória, foi escolhido, entre vários metodos ${ }^{5}$, a análise de Cheng e Tang ${ }^{1}$, por ser mais detalhada e classificar, por uma pontuação, o resultado estético e funcional como "excelente", "bom", "ruim" ou "péssimo", além de considerar a opinião subjetiva do paciente e de seus pais, conforme a Tabela 1.

Foram consideradas apenas as informações pós-operatórias, devido à falta de dados prévios à cirurgia nos prontuários. Comparamos o lado normal ao operado quanto à assimetria facial, posicionamento das orelhas, profundidade dos olhos, aplanamento do malar e pagliocefalia; e ao déficit de mobilidade, rotação e inclinação lateral do pescoço $0^{5,6}$.

A rotação e a inclinação lateral do pescoço foram aferidas com goniômetro de plástico articulado na posição ortostática e comparadas ao lado oposto. A soma final, assim, foi composta pela pontuação acumulada, de acordo com a opinião subjetiva, do déficit de inclinação lateral da cabeça, da rotação lateral do pescoço, da assimetria facial, da inclinação lateral do pescoço, do queloide e da existência de bridas pós-operatórias. O paciente poderia somar um máximo de 21 pontos, sendo que, entre 17 e 21 pontos, o resultado do tratamento era considerado excelente; entre 12 e 16, bom; entre 7 e 11, ruim; e, quando menor que 7 , péssimo ${ }^{1}$.

Dividimos os pacientes entre os que tinham sido submetidos ao tratamento cirúrgico entre 3 e 9 anos de idade e os que tinham 10 ou mais ${ }^{1,3}$, devido ao fato reportado de que pacientes com mais de 10 anos se beneficiam de modo funcional da cirurgia, porém sem melhora significativa da assimetria facial ${ }^{1,3,5,6}$.

\section{RESULTADOS}

A média de idade dos pacientes foi de 11 anos, variando de 3 a 19 anos, sendo 15 do sexo feminino $(71,4 \%)$ e 6 do masculino $(28,6 \%)$. Em 11 pacientes $(52,4 \%)$, verificou-se deformidade no lado direito e em $10(47,6 \%)$, no esquerdo.

Quanto à história clínica, apenas um $(4,7 \%)$ tinha se submetido à tentativa de tratamento conservador com fisioterapia. Quinze pacientes $(71,4 \%)$ nasceram de parto normal e seis $(28,6 \%)$ por cesariana; $11(52,4 \%)$ eram os primogênitos; um (4,7\%) teve uma fratura de clavícula como traumatismo de parto e um (4,7\%) apresentou displasia do quadril como doença associada. Quatro $(19,0 \%)$ tinham um parente de primeiro grau (dois irmãos e dois primos) operados para tratamento de torcicolo muscular congênito.

TABELA 1 - Critérios de avaliação, segundo Cheng e Tang'.

\begin{tabular}{lcccc}
\hline & $\begin{array}{c}\text { Excelente } \\
\text { (3 pontos) }\end{array}$ & $\begin{array}{c}\text { Bom } \\
\text { (2 pontos) }\end{array}$ & $\begin{array}{c}\text { Ruim } \\
\text { (1 ponto) }\end{array}$ & $\begin{array}{c}\text { Péssimo } \\
\text { (0 ponto) }\end{array}$ \\
\hline Déficit rotação $\left(^{(}\right)$ & $<5$ & 6-Oct & Nov-15 & $>15$ \\
Déficit inclinação lateral $\left({ }^{\circ}\right)$ & $<5$ & 6-Oct & Nov-15 & $>15$ \\
Assimetria facial & Nenhuma & Leve & Moderada & Grave \\
Inclinação da cabeça & Nenhuma & Leve & Moderada & Grave \\
Formação de queloide & Nenhuma & Leve & Moderada & Grave \\
Formação de bridas laterais & Nenhuma & Lateral & Lateral, clavicular & Lateral, esternal \\
Opinião subjetiva & Excelente & Bom & Ruim & Péssimo \\
Pontuação final & $17-21$ & Dec-16 & 7-Nov & $<7$ \\
\hline
\end{tabular}


Em relação aos resultados obtidos, quase todos os pacientes classificaram o resultado final do seu tratamento como excelente ou bom (exceto um). Dois pacientes $(9,5 \%)$ tiveram a formação de bridas esternais no seguimento. Cinco pacientes $(23,8 \%)$ apresentaram queloide discreto; um paciente $(4,7 \%)$, moderado; e um paciente $(4,7 \%)$, grave (Figura 2$)$.

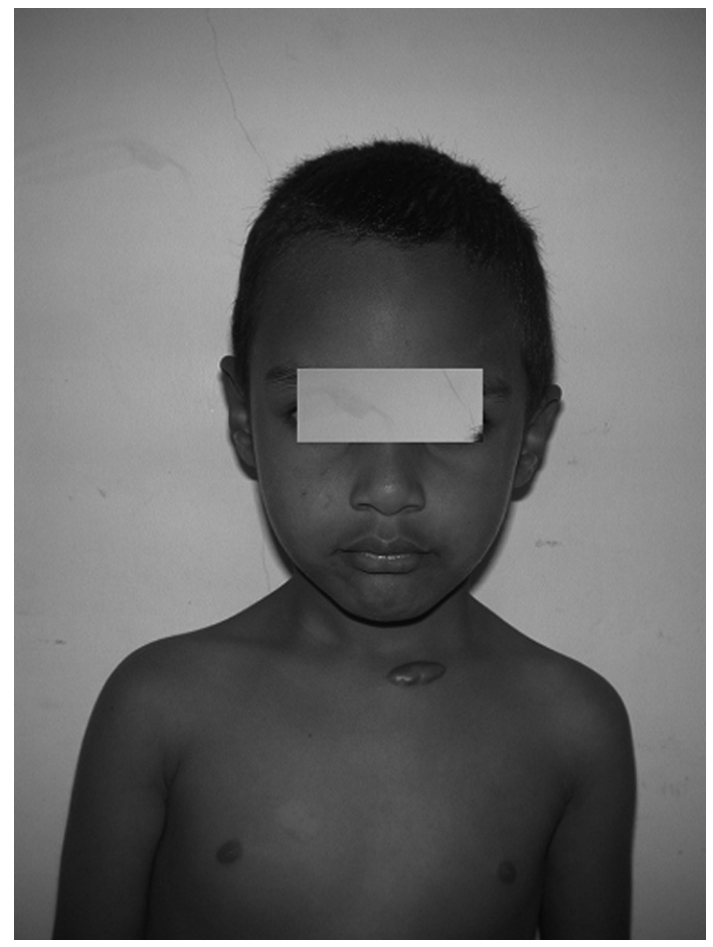

Figura 2

Aspecto clínico do paciente com 11 meses de pós-operatório

A avaliação do resultado estético e funcional revelou que $12(57,1 \%)$ pacientes demonstraram um resultado final excelente, $8(38,1 \%)$ bom, $1(4,7 \%)$ ruim e nenhum paciente teve resultado péssimo, sendo que a melhor pontuação foi observada pelo grupo de crianças submetidas ao tratamento antes dos 10 anos de idade. Estratificando os dados, observou-se que crianças abaixo desta idade tiveram melhores resultados: déficit de inclinação lateral da cabeça $(60 \%$ dos menores de dez anos apresentaram déficit menor que $5^{\circ}$ ); déficit de rotação lateral da cabeça $(100 \%$ das crianças abaixo de dez anos apresentaram déficit menor que $5^{\circ}$ em comparação aos com mais de 10 anos; $20 \%$ permaneceram com déficit de 6 a $10^{\circ}$ de rotação); assimetria facial residual (com $83,3 \%$ dos casos apresentando assimetria leve e $16,7 \%$, nenhuma assimetria residual, ao passo que, nos indivíduos de faixa etária mais elevada, $20 \%$ dos casos tiveram assimetria moderada, $46,6 \%$, assimetria leve, e 33,3\%, não tiveram assimetrias); movimento de inclinação lateral da cabeça (comprometido moderadamente em três pacientes com idade superior a dez anos, ao passo que somente um paciente da faixa etária mais jovem apresentou essa alteração); a limitação da inclinação foi considerada leve em cinco pacientes com faixa etária maior e apenas um paciente do grupo etário mais baixo demonstrou este déficit. Por fim, a pontuação final pós-operatória do paciente também traduz melhores resultados evidenciados em pacientes abaixo de dez anos (Tabela 2).

TABELA 2 - Pontuação final versus faixa etária
no tratamento cirúrgico.
\begin{tabular}{lcccc}
\hline $\begin{array}{l}\text { Idade na } \\
\text { cirurgia }\end{array}$ & $\begin{array}{c}<7 \\
\text { (péssimo) }\end{array}$ & $\begin{array}{c}7-11 \\
\text { (ruim) }\end{array}$ & $\begin{array}{c}12-16 \\
\text { (bom) }\end{array}$ & $\begin{array}{c}>17 \\
\text { (ótimo) }\end{array}$ \\
\hline 3-9 anos & 0 & 0 & 0 & $6(100 \%)$ \\
$10-19$ anos & 0 & $1(6,2 \%)$ & $8(53,3 \%)$ & $6(40 \%)$ \\
\hline
\end{tabular}

\section{DISCUSSÃO}

Verificou-se, na presente série, uma maior incidência em filhos de mães primíparas e pacientes do sexo feminino, compatível com os resultados encontrados por Lidge et al. ${ }^{11}$; porém, não foi possível definir a preponderância do lado direito ou a alta incidência da displasia do quadril descrita na literatura levantada ${ }^{2,8-10,12}$.

No presente trabalho, a média de idade dos pacientes (11 anos) foi muito alta em comparação a trabalhos pesquisados, que reportaram a idade de 1 a 4 anos para a realização de cirurgia, apesar de algumas publicações demonstrarem completa recuperação da assimetria facial em pacientes operados até 12 anos de idade ${ }^{10,13-16}$. Cumpre lembrar também que a grande maioria dos pacientes não foi submetida a nenhuma tentativa de iniciar com tratamento conservador, dados que exaltam as precárias condições socioeconômicas e de acesso ao sistema de saúde dos nossos pacientes.

Observou-se recidiva da deformidade em quatro pacientes $(19,0 \%)$, condizente aos achados de outros autores que variaram de 5 a $33 \%^{13-15,17,18}$. Um paciente foi operado pela primeira vez antes de um ano de idade, outro com quatro anos e os outros dois com nove e dez anos. Todas as recidivas foram resolvidas com a nova intervenção cirúrgica, seguindo o mesmo padrão de abordagem. Ling citou a incidência de $20 \%$ de complicações, sendo o hematoma na substância do músculo liberado a mais comum ${ }^{19}$. Staheli e outros autores, por sua vez, apontaram a possibilidade da ocorrência de bandas laterais entre 38 a 75\% dos casos ${ }^{14,15,18,19}$. Outros autores descreveram a incompleta correção das estruturas com contratura e a inadequada imobilização pós-operatória como fatores que causam deformidade recorrente ${ }^{19}$. Twee $\mathrm{Do}^{8}$ descreveu recidiva das deformidades associada à liberação incompleta da fáscia cervical profunda; segundo este autor, esta seria uma das estruturas mais importantes associada à contratura muscular, devendo, assim, ser liberada completamente - mesmo em planos mais profundos.

Embora exista controvérsia em relação ao tipo de imobilização pós-operatória, concorda-se com os autores que 
opinam a favor do uso do gesso do tipo minerva por algumas semanas, lembrando que nossas crianças são mais velhas e de baixo nível socioeconômico, o que poderia aumentar o índice de recidivas da doença ${ }^{1,19}$. Acredita-se, também, na correção adicional da deformidade com a confecção da cunha corretiva no gesso antes da cicatrização da ferida operatória. Alguns autores ${ }^{5,8,9,20,21}$, no entanto, discordam da utilização de imobilização rígida e orientam a utilização de órteses removíveis, para iniciar uma fisioterapia precoce.

Em relação aos movimentos de rotação e inclinação pós-operatória, os achados do presente trabalho assemelham-se aos de Cheng e Tang ${ }^{1}$, que observaram as melhores médias de movimento nos pacientes operados precocemente. Alguns autores utilizam a liberação bipolar para todos os casos de cirurgia, sem ressecção de nenhum segmento miotendíneo, objetivando a preservação do "V" cervical; porém, esta não foi uma alteração estética importante em nenhum dos nossos $\operatorname{casos}^{5}$.

Apesar da elevada faixa etária dos pacientes submetidos à cirurgia, 18 pacientes $(86 \%)$ permaneceram com leve ou nenhuma assimetria facial, 17 (81\%) com leve ou nenhuma inclinação lateral da cabeça e $1(5 \%)$ apresentou pontuação ruim (11) no resultado final. Este paciente foi operado aos dez anos de idade; contudo, não realizou fisioterapia e exercícios domiciliares orientados durante $o$ período pós-operatório. Ao exame clínico do $15^{\circ}$ mês após o procedimento cirúrgico, apresentava recidiva da deformidade com formação de bridas laterais, sendo indicada a revisão do procedimento.

\section{CONCLUSÕES}

Observou-se que a grande maioria desta série de pacientes com torcicolo congênito submetidos à liberação distal do ECM evoluiu com resultado final bom ou excelente, com baixos índices de complicações.

\section{AGRADECIMENTO}

Agradecemos ao Núcleo de Apoio à Publicação da Faculdade de Ciências Médicas da Santa Casa de São Paulo (NAP-SC) pelo suporte técnico-científico à publicação deste manuscrito.

\section{REFERÊNCIAS}

1. Cheng JC, Tang SP. Outcome of surgical treatment of congenital muscular torticollis. Clin Orthop Relat Res. 1999;(362):190-200.

2. Macdonald D. Sternomastoid tumour and muscular torticollis. J Bone Joint Surg Br. 1969;51(3):432-43.

3. Cheng JC, Wong MW, Tang SP, Chen TM, Shum SL, Wong EM. Clinical determinants of the outcome of manual stretching in the treatment of congenital muscular torticollis in infants. A prospective study of eight hundred and twenty-one cases. J Bone Joint Surg Am. 2001;83-A(5):679-87.

4. Hulbert KF. Congenital torticollis. J Bone Joint Surg Br. 1950;32B(1):50-9.

5. Schneider I, Maçaneiro CH, Pagliosa FR. Torcicolo muscular congênito: resultado do tratamento cirúrgico. Rev Bras Ortop. 1995;30(1/2):11-6.

6. Wei JL, Schwartz KM, Weaver AL, Orvidas LJ. Pseudotumor of infancy and congenital muscular torticollis: 170 cases. Laryngoscope. 2001;111(4 Pt 1):688-95.

7. Porter SB, Blount BW. Pseudotumor of infancy and congenital muscular torticollis. Am Fam Physician. 1995;52(6):1731-6. Review.

8. Do TT. Congenital muscular torticollis: current concepts and review of treatment. Curr Opin Pediatr. 2006;18(1):26-9.
9. Tatli, B. Aydinli N, Caliskan M, Ozmen M, Bilir F, Acar G. Congenital muscular torticollis: evaluation and classification. Pediatr Neurol. 2006;34(1):41-4.

10. Chate RA. Facial scoliosis from sternocleidomastoid torticollis: longterm postoperative evaluation. Br J Oral Maxillofac Surg. 2005;43(5):428-34.

11.Lidge RT, Bechtol RC, Lambert CN. Congenital muscular torticollis; etiology and pathology. J Bone Joint Surg Am. 1957;39-A(5):1165-82.

12.Hummer CD, MacEwen GD. The coexistence of torticollis and congenital dysplasia of the hip. J Bone Joint Surg Am. 1972;54(6):1255-6.

13.Ippolito E, Tudisco C, Massobrio M. Long-term results of open sternocleidomastoid tenotomy for idiopathic muscular torticollis. J Bone Joint Surg Am. 1985;67(1):30-8.

14.Lee EH, Kang YK, Bose K. Surgical correction of muscular torticollis in the older child. J Pediatr Orthop. 1986;6(5):585-9.

15.Minamitani K, Inoue A, Okuno T. Results of surgical treatment of muscular torticollis for patients greater than 6 years of age. J Pediatr Orthop. 1990;10(6):754-9.

16. Tse P, Cheng J, Chow Y, Leung PC. Surgery for neglected congenital torticollis. Acta Orthop Scand. 1987;58(3):270-2. 17.de Chalain TM, Katz A. Idiopathic muscular torticollis in children: the Cape Town experience. Br J Plast Surg.1992;45(4):297-301.

18.Staheli LT. Muscular torticollis: late results of operative treatment. Surgery. 1971;69(3):469-73.

19.Ling CM. The influence of age on the results of open sternomastoid tenotomy in muscular torticollis. Clin Orthop Relat Res .1976;(116):142-8.

20.Canale ST, Griffin DW, Hubbard $\mathrm{CN}$. Congenital muscular torticollis. A long-term follow-up. J Bone Joint Surg Am. 1982;64(6):810-6.

21.Cheng CY, Ho KW, Leung KK. Multiadjustable post-operative orthosis for congenital muscular torticollis. Prosthet Orthop Int. 1993;17(2):115-9.

\begin{tabular}{l}
\hline Correspondência \\
Osmar Avanzi \\
Pavilhão Fernandinho Simonsen do \\
Departamento de Ortopedia/Grupo de \\
Coluna \\
Rua Dr. Cesário Motta Jr, 112 - Vila \\
Buarque \\
CEP: 01221 -020 - São Paulo (SP), Brasil \\
Tel./ Fax: (11) $3224-0122$, ramal: 5552 \\
E-mail: coluna@santacasasp.org.br \\
\hline
\end{tabular}

